\title{
Service Coopetition Under Alliance: A Competitive Dynamics Perspective
}

\author{
Wei-Lun Chang
}

\begin{abstract}
Airline companies join airline alliances to cope with the high level of competition in the airline industry. However, pressure within an alliance is substantial. Few previous studies have investigated alliance selection and dynamic competitive behavior. This study used competitor mapping and awareness-motivation-capability competitive dynamics to analyze airline alliances. The results revealed that alliances compete to recruit airline companies, and the power differences in the alliances are considerable; only a few of the airline companies within the alliance possess power. In addition, we interviewed two senior managers to confirm the results of the analysis. A detailed content analysis was performed to test each hypothesis.
\end{abstract}

Keywords Competitor mapping - Awareness-motivation-capability (AMC) analysis $\bullet$ Competitive dynamics

References available upon request.

W.-L. Chang $(\bowtie)$

Tamkang University, Taipei, Taiwan

e-mail:wlchang@mail.tku.edu.tw 